\section{The Blind Showing The Way To The Sighted}

Jean-Paul Revel, CALTECH

When one speaks of microscopes one usually imagines devices which produce spatially resolved images. So it is most intriguing when a novel application of microscopic techniques permits discoveries not by forming images but by allowing very precise measurements to be made on very small objects. The blind showing the way to the sighted! A recent paper in Nature is a good example of what I speak. It deals with biological motors, which, as cell biologists are learning, are involved in the movement of cells and the movements of organelles within cells. There are a number of different biological motors, but all are molecules which change shape, as they convert the chemical energy derived from the hydrolysis of high energy molecules such as ATP, into mechanical work. As the motor molecules undergo this conformational change and then return to their original state they take a step away from their starting point, dragging along the structures to which they are attached. Using a combination of optical tweezers cum interferometer, Skvoboda, Schmidt, Schnapp and Block of the Rowland Institute in Cambridge, MA and Harvard University, now report that the one of these motors, the molecule called kinesin, about $10 \mathrm{~nm}$ in size, moves by taking 8 $\mathrm{nm}$ steps. As expressed by J.Howard this is "a giant step for kinesin". It is also not so small a step on the path to a detailed understanding of cellular motility, with implications in a large number of areas, like muscle contraction, transport in neurons and other cells, sperm cell and ciliary motility, etc.

The apparatus used in measuring the size of this step is conceptually very simple. As explained by Svoboda et al. it consists of a differential interference contrast (DIC) microscope in which "polarized laser light is introduced at a point just below the objective Wollaston prism. . . the prism splits the light into two beams with orthogonal polarization: they are focused

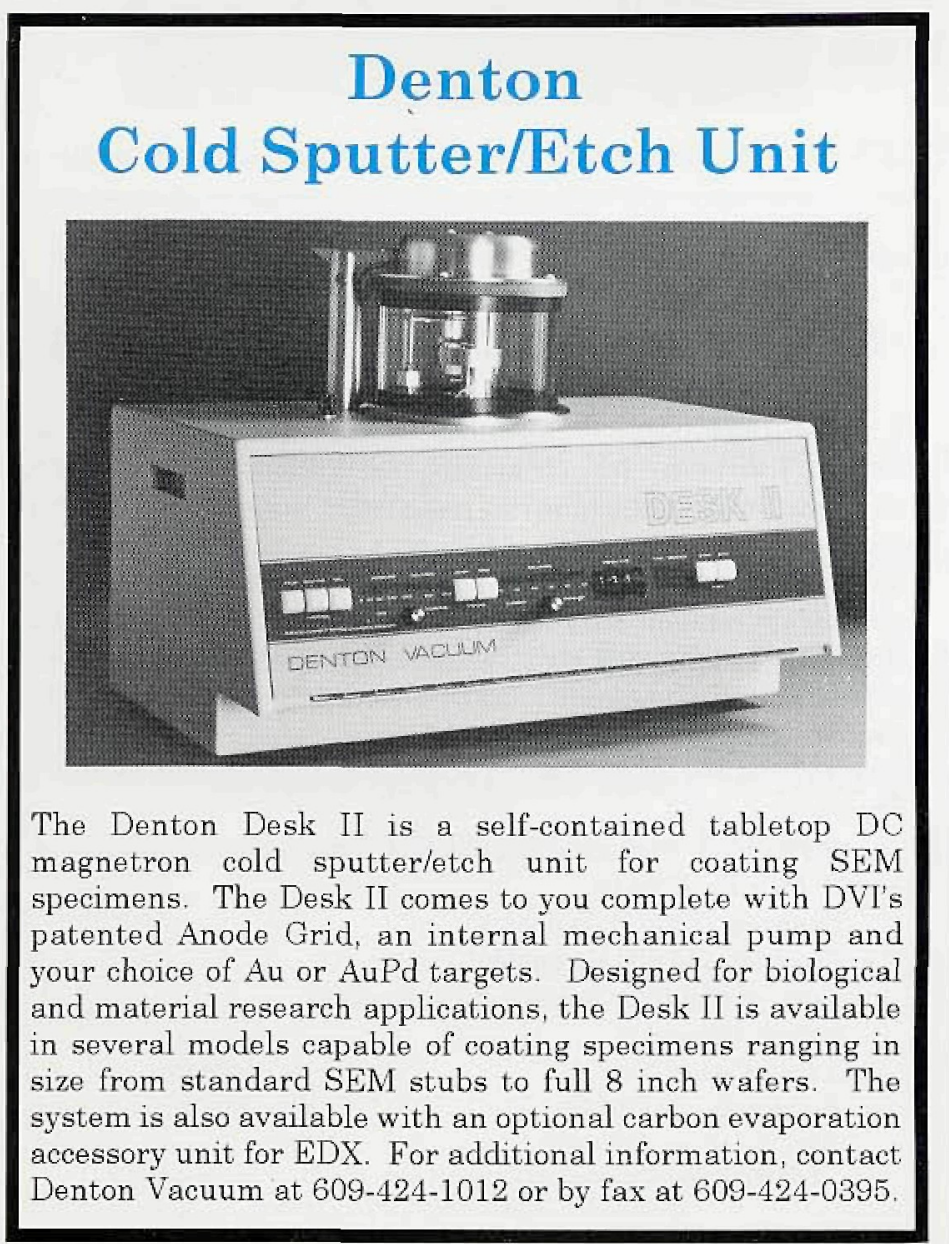

to overlapping diffraction-limited spots at the specimen plan, and together they function as a single optical trap". Small silica beads which were exposed to such low concentrations of kinesin that, on the average, there is only one active kinesin molecule attached to a bead, are placed in the region illuminated by the two spots. Their presence "introduces a relative retardation between the beams". This retardation changes as the bead moves, "passing through zero when the object exactly straddles the two spots. For small excursions (out to $150 \mathrm{~nm}$ ) the output of the detector system is linear with the displacement".

Kinesin molecules attach to, and in the presence of ATP, move along, rails which are formed by microtubules, long cylindrical structures formed of protein molecules, called tubulins. The experiment consists of measuring, in the presence of ATP, the displacement along microtubules of silica beads with a single kinesin attached. Free beads are drawn to the trap center where they settle on and attach to a microtubule (itself attached to a microscope slide), and move along it while ATP is split to ADP untill they somehow become detached, at which time they are drawn back to the center. The beads can be thought of as being linked to two springs which exert forces on it. One of these springs is the optical trap which pulls the bead toward the center; the other is the link between the kinesin molecule and the microtubule which pulls along in a direction parallel to the microtubule. Because the whole system is thus under tension, one can more readily detailed features of the movement, which otherwise would be hidden in the noise due to brownian (etc.) motion.

Statistical analysis of the data obtained under a variety of conditions shows that the kinesin molecules move in step wise manner along the microtubule, with each step $8 \mathrm{~nm}$ in size. When the concentration of ATP is not limiting a single kinesin molecule can move at a rate of $3-500 \mathrm{~nm} / \mathrm{s}$, imagine $0.5 \mathrm{micron} / \mathrm{s}$ !! With the laser power set relatively high $(58 \mathrm{~mW}$ at the specimen plane) the tweezers exerted a force varying from 0 (at the center) to about $5 \mathrm{pN}$ at the edge. The beads in the center move rapidly as we have just indicated but at the edges of the trap, where the latter "pulls" hardest, most slow or become stuck. Some of the beads nevertheless can escape, i.e. were able to exert forces greater than $5 \mathrm{pN}$ and thus win the tug of war with the optical tweezers. So we have a measure of the strength of champion kinesin molecules. When the ATP concentration is reduced, the time between steps was found to increase. This would be compatible with the idea that when one molecule of ATP is used one has to wait for another to attach before the cycle can repeat. This suggests that one molecule of ATP is used per step, although this still has to be proved formally. Not only do we know how big a step the molecular tug kinesin can take, we learn how fast the tug can go, how hard it pulls and get clues about how much energy it consumes. Not bad for a "non imaging" microscope!

\section{References:}

1. Svoboda, K., Schmidt, C.F., Schnapp, B.J., and Block, S.M. (1993). Direct Observation of Kinesin Stepping by Optical Tapping Interferometry. Nature..365, 721-727

2. Howard, J. (1993) One Giant Step for Kinesin. Nature, 365, 696-697

New \& Affordable

OPIICAL MICROSCOPES

$$
\$ 125 \text { TO } \$ 1,450
$$

\author{
M.E. TAYLOR ENGINEERING INC. \\ 21604 Gentry Lane - Brookeville, MD 20833 \\ Tel.: (301)774-6246 - Fax: (301)774-6711
}

